resulted in an odds ratio of 1.14 (95\% CI of 0.95-1.35) compared to individuals unexposed to PAHs; results were similar at substantial levels of exposure. Considering workers exposed to only a single profile of PAH depending on the combustion product, results were: PAH from wood [0.88 (0.35-2.21)], from coal [0.96 (0.52-1.78)], from petroleum [1.04 (0.86-1.25)], and from other sources [1.84 (1.04-3.25)]. Exposure to benzo (a) pyrene resulted in an odds ratio of 1.31 (1.06-1.63).

Conclusions Results suggested excess risk associated with exposure to benzo (a)pyrene and to PAHs arising from cooking fumes, plastic, and rubber; but not for PAH exposures derived from wood, coal, and petroleum combustion products. These results are partly in line with previous reports of highly exposed workers, though exposure levels would not have been as high in our population-based study.

\section{CANCER INCIDENCE AND MORTALITY IN AGRICULTURAL COHORTS IN THE AGRICOH CONSORTIUM}

${ }^{1}$ R E Denholm, ${ }^{2}$ Beane Freeman, ${ }^{1}$ Schüz, ${ }^{2}$ Alavanja, ${ }^{3}$ MacFarlane, ${ }^{3}$ Sim, ${ }^{4}$ Lebailly, ${ }^{5}$ Baldi, ${ }^{6}$ Kjaerheim, ${ }^{6}$ Langseth, ${ }^{7}$ Nordby, ${ }^{8}$ Park, ${ }^{8}$ Yoo, ${ }^{9}$ Keifer, ${ }^{10}$ Waring, ${ }^{1}$ Ferro, ${ }^{11}$ Douwes, ${ }^{12}$ Hoppin, ${ }^{13}$ Kromhout, ${ }^{14}$ Schenker, 'Leon. International Agency for Research on Cancer, Lyon, France; ${ }^{2}$ National Cancer Institute, Bethesda, Maryland, United States of America; ${ }^{3}$ Monash University, Victoria, Australia; ${ }^{4}$ Université de Caen Basse-Normandie, Caen, France; ${ }^{5}$ Universite Bordeaux 2, Bordeaux, France; ${ }^{6}$ The Cancer Registry of Norway, Oslo, Norway; "National Institute of Occupational Health, Oslo, Norway; ${ }^{8}$ Seoul National University College of Medicine, Seoul, South Korea; ${ }^{9}$ National Farm Medicine Center, Marshfield, Wisconsin, United States of America; ${ }^{10}$ Essentia Institute of Rural Health, Duluth, Missesota, United States of America; "Massey University, Wellington, New Zealand; ${ }^{12}$ National Institute of Environmental Health Sciences, North Carolina, United States of America; ${ }^{13}$ Utrecht University, Utrecht, Nederland; ${ }^{14}$ University of California at Davis, Davis, California, United States of America

\subsection{6/oemed-2013-101717.106}

Objectives Farmers have different cancer incidence and mortality patterns than the general population, with excesses of some cancers (e.g., lymphoma, multiple myeloma, brain, skin and prostate), and lower risk of others (e.g., lung). AGRICOH, a consortium of 27 cohort studies in agricultural populations, was formed to investigate the impact of specific occupational, environmental and lifestyle factors on health outcomes in farming populations. This study aims to describe the cohort-specific cancer incidence and mortality rates in AGRICOH studies with cancer information.

Methods Outcome data have been obtained from linkage to cancer and mortality registries. Person-years of follow-up contributed by each participant will be calculated and grouped into 5 -year age intervals and calendar years. Crude and age- and gender- standardised incidence and mortality rates will be estimated for each cohort, for all cancers, individual cancer sites and, where possible, specific histological subtypes. Rates will be stratified by participant's relationship to the farm (farmer, spouse) and primary commodity produced (crop, livestock or both).

Results Seven AGRICOH cohorts with cancer data have agreed to participate $(\mathrm{n}=315,298)$. These cohorts represent Australia (Pesticide Exposed Workers \& Victorian Grain Farmers), France (Agriculture \& Cancer), Korea (Korean Multi-Center Cancer), Norway (Cancer in the Norwegian Agricultural Population), and USA (United States Agriculture Health Study \& The Marshfield Epidemiology Study Area Farm Cohort). Target populations vary between studies and include active and retired agricultural workers, farm owners and their families, and agricultural groups exposed to specific agents.
Conclusions Comparing and contrasting patterns of cancer incidence and mortality in studies from around the world with different agricultural practices and populations will generate hypotheses for future data-pooling projects. In particular, the study will identify cancers of high priority in agricultural workers and provide hypotheses on potential causes for differences in rates between populations, or on common exposures that might contribute to similarities.

\section{Session: N. Noise \& hearing}

\section{NOISE EXPOSURE AND HEARING LOSS AMONG NAVAL PERSONNEL}

K I H Irgens-Hansen, B E M Moen, M B Bråtveit, C Hauge, E Sunde. University of Bergen, Bergen, Norway

\subsection{6/oemed-2013-101717.107}

Objectives In a questionnaire conducted by The Royal Norwegian Navy in $2002 / 2003,38 \%$ of the personnel reported to be much/very much exposed to noise and $24 \%$ reported reduced hearing. Based on these findings, further investigation on the working conditions on board naval vessels and mapping NIHL among the personnel on board was initiated. A part of this study is to compare noise levels and occurrence of hearing loss on large vessels like frigates and smaller vessels like coastal corvettes.

Methods Noise levels in areas were measured on three costal corvettes (CC) and three frigates (F) using Brüel \& Kjær Hand-held Analyzer Type 2250. All personnel working on frigates and coastal corvettes were invited to audiometric testing, using Interacoustics AD226. NIHL was classified as $\geq 25 \mathrm{~dB}$ in at least one ear at 3000,4000 or $6000 \mathrm{~Hz}$. They completed a questionnaire concerning noise exposure and background information.

Preliminary Results Noise levels in cabins range from $68.5 \mathrm{~dB}$ (A) to $85.5 \mathrm{~dB}(\mathrm{~A})$ in $\mathrm{CC}$ and from $42.1 \mathrm{~dB}(\mathrm{~A})$ to $80 \mathrm{~dB}(\mathrm{~A})$ in $\mathrm{F}$. 157 persons were currently or previously working on $\mathrm{F}$ and 91 persons were currently or previously working on CC. The prevalence of NIHL among personnel in CC was $40.7 \%$ and $28.7 \%$ among personnel in $\mathrm{F}$ (Pearson Chi-Square test, $\mathrm{P}=0.053$ ). Mean age for CC was 32 and for F 28 years. When adjusting for age using logistic regression no differences in NHIL among the two vessel groups were found.

Conclusions The CC has higher measured noise levels than the F. Our analysis shows a higher prevalence of NIHL among personnel currently or previously working on CC compared to F. When adjusting for age the effect disappears. This may indicate that the NIHL found was not related to vessel group.

\section{HEARING SURVEILLANCE IN THAI FORKLIFT DRIVERS WITH ENVIRONMENTAL MEASUREMENT}

W K Karnjanakantorn. Bangkok Hospital Group, Bangkok, Thailand

10.1136/oemed-2013-101717.108

Background Noise is one of physical hazards found in forklift drivers, especially with diesel engine. Thailand's 2010 regulation on the control of noise at work is not more than $85 \mathrm{dBA}$ per 8 hour-TWA. 
Objectives To survey the hearing problem and noise exposure of forklift drivers in a factory.

Methods A cross-sectional survey was conducted during 20112012 among the workers. Data was collected through periodic examination and environmental measurement. A total of 21 people participated in the study ( 20 males, 1 female).

Results The workers were 29-48 years of age and their working years were between 9 to 20 years with the average working time of 40 hours per week. The report of abnormal audiogram was 33.3\% (7 people) and one case was later excluded after the repeated test was normal. Two cases were diagnosed with moderate to severe sensorineural hearing loss while the others (5 people) were diagnosed with mild SNHL. All of them were not concerned about ear protective equipment, nor did they utilize such equipment. The environmental measurement was done by sound level meter; Noise Dosimeter. Time weighted average level (8-hr-TWA) was still under the regulations, which were between 80.38 to $85.61 \mathrm{dBA}$.

Conclusion Hearing conservative program should be provided to high risk workers even though the noise induced hearing loss was not diagnosed. The periodic examination is important to compare with baseline audiogram.

\section{WORK-RELATED NOISE INDUCED HEARING LOSS CASES REPORTED BY PHYSICIANS TO THE NORWEGIAN REGISTRY OF WORK-RELATED ILLNESSES: DATA FROM 2005-2009}

${ }^{1} Y$ S Samant, ${ }^{2}$ Lysberg, ${ }^{2}$ Landrø, ${ }^{2}$ Wergeland. 'Stjordal, Norway; ${ }^{2}$ Norwegian Labour Inspection Authority, Trondheim, Norway

\subsection{6/oemed-2013-101717.109}

Background This study provides an epidemiological profile of hearing loss cases reported to the Norwegian Labour Inspectorate (NLI) and also a distribution of cases by the notifying physician type.

Materials and Methods The study is based on obligatory physician notifications of work-related illnesses to the Norwegian Labour Inspectorate. These physician reports are the basis for the Registry of Work-related illnesses. We extracted NIHL data from this registry based on the ICD-10 codes for the period 2005-2009 (5-year period). We also obtained employment data from Statistics Norway by trade sector, gender and age. We then estimated the average number of cases reported in the period 2005-2009. Next we estimated the incidence rates for the reported cases by gender, age and trade sector. We also computed descriptive statistics for occupation and the type of notifying physician.

Results In the 5-year period, a total of 7888 cases of NIHL were reported to the NLI. On average 1577 cases of NIHL were reported. $96 \%$ of these cases were men. Incidence of reported work-related NIHL was estimated to be 66/ 100000 workers. The incidence for reported NIHL cases was respectively 6 and 120/100 000 workers for women and men.

The highest incidence was found in age group 55-66. Cases reported from manufacturing, electricity, gas, steam, construction and mining sectors were found to have the highest incidence rates.

Occupational health physicians reported $85 \%$ of all the NIHL cases while hospital and general physicians reported $7 \%$ and $4 \%$ of the cases respectively.

Conclusions Work-related NIHL remains an extensive problem, yet an under-profiled problem in Norway. Targeted interventions toward vulnerable groups to reduce noise exposure to vulnerable groups are necessary.

The registry of work-related illnesses is not ideal in detecting cases of NIHL because of extensive underreporting and remedial measures ought to be taken to address this issue.

\section{EFFECTS OF WHOLE BODY VIBRATION ON HEARING LEVEL SHIFTS}

${ }^{1}$ S A M N Moussavi-Najarkola, ${ }^{2}$ Khavanin, ${ }^{3}$ Mirzaei, ${ }^{4}$ Salehnia. ${ }^{1}$ College of Health, Shahid Beheshti University of Medical Sciences (SBUMS), Tehran, Iran; ${ }^{2}$ Department of Occupational Health, School of Medical Sciences, Tarbiat Modares University, Tehran, Iran; ${ }^{3}$ Department of Occup. Health, Faculty of Health, Zahedan University of Medical Science, Zahedan, Iran; ${ }^{4}$ Department of Anatomical Sciences, School of Medical Sciences, Tarbiat Modares University, Tehran, Iran

\subsection{6/oemed-2013-101717.110}

Background and Aim Adverse effects of occupational whole body vibration (WBV) on hearing are yet unknown. The aim of the research was to survey effects of whole body vibration on hearing shifts.

Methods Male adult New Zealand White rabbits were divided into two groups include control group $(\mathrm{n}=6$, not exposed to whole body vibration) and vibration group ( $\mathrm{n}=6$, exposed to whole body vibration in the Z-axis at $4 \mathrm{~Hz}$ and $1.0 \mathrm{~ms}^{-2}$ r.m.s for 8 hours per day in 5 consecutive days by putting their box on a vibrating platform). DPOAEs were measured hearing shifts in vibration group in days: zero (before exposure to vibration) as baseline, eighth (an hour after exposure to vibration) as temporary threshold shifts, and tenth (48 hours after exposure to vibration) as permanent threshold shifts. Similarly, DPOAEs were also examined hearing shifts in control group in days zero, eighth, tenth. The gathered data analysed by independent-samples T Test.

Results Increased mean DPOAEs amplitudes were observed in frequencies $375.00,562.50,750.00,1125.00,1312.50$, 2062.50, 2625.00, 3937.50, 5437.50, 6562.50 Hz respectively in vibration group. There were no any significant differences between mean DPOAEs amplitudes in days zero, eighth and tenth at all frequencies $(\mathrm{p}=0.073)$.

Conclusion The exposure to vibration significantly led to enhanced mean DPOAEs amplitudes at all frequencies at low frequencies rather than at high frequencies. Thus, this study showed that vibration exposure only could not cause temporary or permanent threshold shifts. Increased DPOAEs amplitudes could be probably attributed to normal outer and destroyed inner hair cells.

\section{Session: 0. Biomarker}

\section{PRODUCTION OF IL-10, TNF AND IL-12 BY PERIPHERAL BLOOD MONONUCLEAR CELLS IN MEXICAN WORKERS EXPOSED TO A MIXTURE OF BENZENE-TOLUENE-XYLENE}

${ }^{1} \mathrm{~L}$ H Haro-Garcìa, ${ }^{2} \mathrm{C}$ A J P Juárez-Pérez, ${ }^{2} \mathrm{G}$ A M Aguilar-Madrid, ${ }^{2} \mathrm{~N} V \mathrm{Z}$ Vélez-Zamora, ${ }^{3} S$ M Muñoz-Navarro, ${ }^{4}$ R C H S Chacón-Salinas, ${ }^{2}$ C R G B González-Bonilla, ${ }^{4}$ C R I H IturbeHaro, ${ }^{4}$ I E G Estrada-García, ${ }^{2} V$ H Borja-Aburto. 'Universidad Autònoma de la Ciudad de Mèxico, Delegaciòn Cuauhtèmoc, $C$ d. de Mèxico, Mexico; ${ }^{2}$ Instituto Mexicano del Seguro Social, Mexico City, Mexico; ${ }^{3}$ Universidad de la Frontera, Temuco, Chile; ${ }^{4}$ Instituto Politécnico Nacional, Mexico City, Mexico

10.1136/oemed-2013-101717.111 